\title{
The relationship between star formation rates and mid-infrared emission in galactic disks ${ }^{\star}$
}

\author{
H. Roussel ${ }^{1}$, M. Sauvage ${ }^{1}$, L. Vigroux ${ }^{1}$, and A. Bosma ${ }^{2}$ \\ 1 DAPNIA/Service d'Astrophysique, CEA/Saclay, 91191 Gif-sur-Yvette Cedex, France \\ 2 Observatoire de Marseille, 2 place Le Verrier, 13248 Marseille Cedex 4, France
}

\author{
Received 2 February 1999 / Accepted 19 March 2001
}

\begin{abstract}
The $\mathrm{H} \alpha$ and mid-infrared mean disk surface brightnesses are compared in a sample of nearby spirals observed by ISOCAM. This shows that, in spiral disks, dust emission at 7 and $15 \mu \mathrm{m}$ provides a reasonable star formation tracer. The fact that the 15 to $7 \mu \mathrm{m}$ flux ratio is nearly constant in various global exciting conditions indicates a common origin, namely the aromatic infrared band carriers, and implies that at these wavelengths, dust emission from the disks of normal galaxies is dominated by photodissociation regions and not by HiI regions themselves. We use this newly-found correlation between the mid-infrared and the $\mathrm{H} \alpha$ line to investigate the nature of the link between the far-infrared $(60$ and $100 \mu \mathrm{m})$ and $\mathrm{H} \alpha$. Although the separation of the central regions from the disk is impossible to achieve in the far-infrared, we show that a circumnuclear contribution to the dust emission, having no equivalent counterpart in $\mathrm{H} \alpha$, is most likely responsible for the well-known nonlinearity between far-infrared and $\mathrm{H} \alpha$ fluxes in spiral galaxies. We derive a calibration of 7 and $15 \mu \mathrm{m}$ fluxes in terms of star formation rates from a primary calibration of $\mathrm{H} \alpha$ in the literature, and also outline the applicability limits of the proposed conversion, which should not be blindly extrapolated to objects whose nature is unknown.
\end{abstract}

Key words. galaxies: spiral - galaxies: ISM - stars: formation - infrared: ISM

\section{Introduction}

Whether mid-infrared emission can be considered a reliable tracer of the massive stellar content of normal and isolated spirals is still unclear. The accepted interpretation of mid-IR spectra of galaxies (see the review by Puget \& Léger 1989; Désert et al. 1990) is that they consist primarily of a composite of a featureless continuum and of a family of aromatic bands, the so-called unidentified infrared bands (UIBs). The continuum emission is attributed to very small grains, VSGs (Désert et al. 1990), of which little is known, while various carbonaceous materials have been suggested as candidates to carry the UIBs, among which the PAH model (polycyclic aromatic hydrocarbons) of Léger \& Puget (1984) has been a long-time favorite. However, the recent work of Boulanger et al. (1998a) indicates that UIB carriers are likely aggregates of several hundred atoms rather than macro-molecules. It is important to realize that under most radiation field conditions,

Send offprint requests to: H. Roussel, e-mail: hroussel@cea.fr

* Based on observations with ISO, an ESA project with instruments funded by ESA Member States (especially the PI countries: France, Germany, The Netherlands and the UK) and with the participation of ISAS and NASA. both components are out of thermal equilibrium and undergo large temperature fluctuations of several hundred K.

Sturm et al. (2000) have provided a census of the continuum emission and of emission features (UIBs and ionic lines) found from 2.4 to $45 \mu \mathrm{m}$ in typical starburst galaxies, and which are potentially present in our data as well. Emission from the envelopes of cold stars can also contribute in the $7 \mu \mathrm{m}$ bandpass, but it is negligible in our sample, except possibly in the disks of two $\mathrm{S} 0 / \mathrm{a}-\mathrm{Sa}$ galaxies.

This dual nature of the mid-infrared emission (produced mainly by two dust phases, UIB carriers and VSGs) makes the existence of a direct link with massive stars unlikely. A further complication is that even if both species are predominantly heated by high energy radiation, their excitation by optical and near ultraviolet photons may be significant in environments where old stellar populations dominate. Indeed, aromatic bands are ubiquitous in the diffuse interstellar medium (Giard et al. 1989; Mattila et al. 1996) and are also observed in regions where the ultraviolet radiation density is insufficient to account for their heating (Sellgren et al. 1990; Boulade et al. 1996; Uchida et al. 1998, 2000). When observing extragalactic objects, emission arising in star forming regions is mixed 
with that arising in the interstellar medium associated with more evolved stars. Therefore, the accuracy with which mid-infrared emission traces star formation should in principle depend on the balance between these two heating sources. This can be checked either by investigating spatially resolved galaxies, or by building global energy budgets. As we will show later, the flux fraction due to heating by old stellar populations is generally quite small in spiral galaxies, as already noted by Lemke et al. (1998).

An attempt to link the mid-infrared emission of galaxies with recent star formation is, however, encouraged by the following fact: resolved observations of individual regions in our Galaxy have revealed that aromatic bands are closely associated with the photodissociation shells and surfaces of molecular clouds in the vicinity of Hit regions or hot stars, while the VSG continuum strongly peaks inside HiI regions (Cesarsky et al. 1996; Tran 1998; Verstraete et al. 1996). Given that both types of sites are intimately linked with the presence of massive stars, a strong coupling between mid-infrared emission and present-day star formation could exist.

An invaluable advantage of infrared observations over optical recombination lines or the ultraviolet continuum resides in their much lower sensitivity to interstellar extinction, thus providing insights into obscured star forming regions. Besides, if it is confirmed that young stars are the major heating source of dust emitting at 7 and $15 \mu \mathrm{m}$, mid-infrared fluxes could provide a more acceptable star formation tracer than far-infrared fluxes, since the latter have been shown to contain a cirrus component (Helou 1986) which dominates the mean emission from morphological types Sa to Sc (Sauvage \& Thuan 1992) and is responsible for a strong non-linearity in the correlation between far-infrared and $\mathrm{H} \alpha$ fluxes. A vast literature covers the advantages and limitations of interpreting far-infrared emission as a star formation indicator, and includes for instance Lonsdale-Persson \& Helou (1987), Devereux \& Young (1990) and Smith \& Harvey (1996). A summary of the issues in question can be found e.g. in Sauvage \& Thuan (1992) or in Kennicutt (1998a).

On the other hand, since survey programs performed with ISO have focussed on observations at 7 and $15 \mu \mathrm{m}$, investigating the relationship between the emission in these bandpasses and star formation rates would be very helpful for their physical interpretation.

To tackle this question, we use a sample of 69 galaxies observed by ISOCAM in two broadband filters centered at 7 and $15 \mu \mathrm{m}$. All the galaxies being nearby, the achieved spatial resolution is sufficient to delineate distinct structural entities (spiral arms, giant HiI complexes, the circumnuclear concentration, etc.). Distances to the sample galaxies range from 4 to $60 \mathrm{Mpc}$, which translates into linear resolutions of $110 \mathrm{pc}$ to $1.7 \mathrm{kpc}$ at $7 \mu \mathrm{m}$ (full width at half maximum of the point spread function). This allows a clear differentiation of the mid-infrared properties of central regions and disks, a study described in Roussel et al. (2001a and b, hereafter Atlas and Paper I). In this paper, we use this advantage to restrict ourselves to the study of galactic disks. Our motivation for that is manifold:

(1) The mid-infrared colors of galaxies in our sample are relatively uniform in the disks, while the circumnuclear regions can show strong $15 \mu \mathrm{m}$ emission excesses, suggesting the existence of a different thermodynamical state of dust in the central parts of galaxies.

(2) Since we want to establish a calibration of mid-infrared fluxes in terms of star formation rates, we need to compare them with a direct primary star formation tracer and so far, few of them sample equally well the disk and the nuclear regions of galaxies, mainly because of extinction.

(3) It has been shown (Kennicutt 1998a) that star formation processes and physical conditions prevailing in nuclei are widely different from those of disks.

In Sect. 2 we present our sample and the methods used to collect the photometric information needed for our analysis. Section 3 demonstrates the validity of mid-infrared fluxes as star formation tracers in galactic disks. In Sect. 4 we discuss the applicability limits of our calibration and the implications of our findings on the interpretation of the far-infrared emission of galaxies.

\section{The galaxy sample and photometric data}

The sample of spiral galaxies considered here is made from the partial merging of five ISOCAM programs:

- Camspir (PI: L. Vigroux) which mapped nearby very large spiral galaxies extensively observed in other interstellar tracers, allowing detailed spatial analyses.

- Cambarre (PI: C. Bonoli) which mapped barred spiral galaxies, selected to span the variety of bar and Hubble types and a large range of infrared luminosities.

- Resolved spirals from the complete survey of the Virgo program (PI: J. Lequeux; see Boselli et al. 1998).

- Non-Seyfert spirals, with no strong signs of tidal interaction, from the Sf_glx program (PI: G. Helou; see Dale et al. 2000) which selected galaxies sampling the IRAS color-color diagram (Helou 1986).

- Identically chosen spirals from the Galir program (PI: T. Onaka) that aimed at constructing infrared spectral energy distributions of normal galaxies, in preparation for the Japanese mission IRTS.

The resulting sample comprises 69 galaxies spanning the whole de Vaucouleurs spiral sequence from S0/a to Sdm. All were observed in raster mode in the two filters LW2 centered at $7 \mu \mathrm{m}(5-8.5 \mu \mathrm{m})$ and LW3 centered at $15 \mu \mathrm{m}$ $(12-18 \mu \mathrm{m})$, with a pixel size of $3^{\prime \prime}$ or $6^{\prime \prime}$. General information and a deeper discussion of the mid-infrared properties of these galaxies can be found in Paper I, together with the spectra between 5 and $16 \mu \mathrm{m}$ observed in five of them. The detailed description of data reduction appears in the Atlas, that also presents the $7 \mu \mathrm{m}$ maps. We simply note here that we processed all maps in a homogeneous way, including those already published. 


\subsection{Ho data}

Since the goal of this paper is to assess the reliability of mid-infrared emission as a star formation tracer, we need to collect data on a primary indicator. Recombination lines, which trace the existence of massive stars, are an obvious choice: well-established calibrations in terms of star formation rate (SFR) exist, and their production by post-AGB stars, as seen in ellipticals (Binette et al. 1994), is negligible in star-forming galaxies (Kennicutt 1998a).

We therefore searched the literature for integrated $\mathrm{H} \alpha$ photometry. In addition, some $\mathrm{H} \alpha$ maps were kindly made available to us by J.A. García-Barreto for NGC 1022 and NGC 4691 (published in García-Barreto et al. 1996); T. Storchi-Bergmann for NGC 1097 and NGC 1672 (published in Storchi-Bergmann et al. 1996); M. Naslund for NGC 1365 (published in Lindblad 1999, courtesy of S. Jörsäter, M. Naslund and J. J. Hester); C. Feinstein for NGC 7552 (data published in Feinstein 1997); M. W. Regan via D. Reynaud for NGC 1530 (published in Regan et al. 1996); S. D. Ryder via A. Vogler for NGC 5236 (published in Ryder et al. 1995; Vogler et al. 2001); F. Viallefond for NGC 5457. Some of these maps have been corrected for a spatial gradient, using the background, or for an over-subtraction of the continuum emission, using $I$-band images. In some cases, we also performed the fluxcalibration, using data from the literature inside various apertures.

Concerning galaxies for which we have no map, the bulk of the data comes from Young et al. (1996). We note that this reference provides $(\mathrm{H} \alpha+[\mathrm{NII}])$ fluxes systematically higher than those of Kennicutt \& Kent (1983) for the galaxies in common (by a factor ranging from about one to two). We thus preferred to adopt data from Young et al. (1996) or other references, but for six of our galaxies, they were taken from Kennicutt \& Kent (1983); we have corrected them by a factor 1.16, following Kennicutt (1998b)'s prescription, and we have checked that the optical diameter of these galaxies is less than or comparable to the $\mathrm{H} \alpha$ aperture used (except for VCC 2058: the optical size and $\mathrm{H} \alpha$ aperture are respectively $4.27^{\prime}$ and $3^{\prime}$ ).

Since the central regions of most galaxies in our sample stand out in the mid-infrared as having different properties from the disk (see Paper I and the Atlas) and as the contamination of $\mathrm{H} \alpha$ fluxes by nuclear regions can be significant in galaxies harboring non-stellar activity or starburst, and for all the reasons emphasized in Sect. 1, we chose to exclude the central regions from both midinfrared and $\mathrm{H} \alpha$ measurements. For this purpose, we used matched apertures which were dictated by the available $\mathrm{H} \alpha$ data in the literature. We aimed at subtracting circumnuclear fluxes of sufficiently large a region to match that region inside which most of a possible $15 \mu \mathrm{m}$ excess is located. It was straightforward to achieve this when we could directly perform measurements on $\mathrm{H} \alpha$ maps. However, this could not be achieved in practice for all galaxies, due to the difficulty of finding suitable $\mathrm{H} \alpha$ data. When such nuclear data are not available or were mea- sured only inside an aperture significantly smaller than the size of the circumnuclear region in the mid-infrared (this concerns 8 galaxies out of 44), we checked that the circumnuclear $F_{15} / F_{7}$ color is low (below 1.2), i.e. shares the main characteristic of disks. This ensures that we are not introducing a strong bias, because the color indicates that the star formation process and extinction should be close to those found in disks. The aperture used by Pogge (1989) (from whom the central $\mathrm{H} \alpha$ fluxes of 8 galaxies are obtained) is not given explicitly, but could be estimated from his H $\alpha$ images, except for VCC 460 and VCC 857, for which we have assumed that it is equal to the infrared size of the central region. This could be problematic in the case of VCC 460 , whose central color is high $\left(F_{15} / F_{7}=2.55\right)$. However, all these uncertainties remain a negligible source of error with respect to the extinction correction.

Most $\mathrm{H} \alpha$ measurements (at $6563 \AA$ ) include the two neighboring [NII] lines (the most intense at $6583 \AA$ and another one at $6548 \AA$ ). We applied the same correction as Kennicutt (1983) for average [NII] contamination in disk HiI regions (25\% of the total flux). The $[\mathrm{NII}] / \mathrm{H} \alpha$ ratio is in general higher in central regions than in disk His regions (e.g. Brand et al. 1981), but we have removed central $([\mathrm{NII}]+\mathrm{H} \alpha)$ fluxes, and ratios in disks are little dispersed (Kennicutt \& Kent 1983).

We also made use of the value given by Kennicutt (1983) for average and uniform extinction in the $\mathrm{H} \alpha$ line (expected to be the major source of uncertainty, since it amounts to $1.1 \mathrm{mag})$. It is clear that a uniform extinction correction is in principle very far from the true correction that should be applied. However, we can first expect that regions where the extinction most significantly departs from this value are located in the central parts of galaxies, which we have excluded from the present analysis. Second, it is on $\mathrm{H} \alpha$ data corrected in this way that SFR calibrations are built. And third, apart from observationally deriving the extinction in each object, it is not possible to define a correction scheme (e.g. based on Hubble type, or on global Balmer decrement) that does not introduce as much uncertainty and bias as it supposedly removes. We therefore choose to confine ourselves to this uniform correction scheme, bearing in mind that our conclusions are relative to this method of correcting $\mathrm{H} \alpha$ data in order to estimate the SFR.

Control on the bias that we introduce thereby can be found in an examination of the $\mathrm{H} \alpha$ to $15 \mu \mathrm{m}$ flux ratio (since we apply a uniform correction on the $\mathrm{H} \alpha$ data, it makes no difference here whether these are corrected or not). The variation of the ratio of $\mathrm{H} \alpha$ to $15 \mu \mathrm{m}$ fluxes as a function of the inclination (estimated from kinematical data or, if unavailable, from the ratio of major to minor isophotal diameters) is a pure scatter diagram. We also checked that separating our sample into two morphological classes (22 S0/a-Sb and $20 \mathrm{Sbc}-\mathrm{Sdm}$ ) did not result in significant a difference in the $\mathrm{H} \alpha$ to $15 \mu \mathrm{m}$ flux ratio: the logarithmic means in the two subsamples are respectively $-0.67 \pm 0.18$ and $-0.66 \pm 0.21$. Finally, no trend can be seen in a plot of the $\mathrm{H} \alpha$ to $15 \mu \mathrm{m}$ flux ratio versus 
the size-normalized $\mathrm{H} \alpha$ flux (see Sect. 3 for a definition of this quantity). Since the $15 \mu \mathrm{m}$ emission is much less prone to absorption, we are therefore confident that no systematic variation in the extinction affects the $\mathrm{H} \alpha$ data. Furthermore, the values of $F_{15} / F(\mathrm{H} \alpha)$ in our sample are all compatible with moderate absorption if compared with the values observed in M51 (Sauvage et al. 1996).

Galactic extinction was corrected using the blue absorptions listed in the RC3 together with the extinction curve of Cardelli et al. (1989). The galaxies for which suitable $\mathrm{H} \alpha$ data were found are listed in Table 1 with their total and central fluxes.

\subsection{Mid-IR photometry}

We have measured total fluxes as explained in the Atlas, and central fluxes inside the same aperture as that used for $\mathrm{H} \alpha$, in images treated with an algorithm designed to correct for dilution effects, also described in the Atlas. The disk flux is then the difference between the total flux and the corrected nuclear flux. The resulting central flux fractions which have been removed and disk fluxes are given in Table 1.

As already mentioned, the aperture of $\mathrm{H} \alpha$ measurements matches reasonably well the size of the circumnuclear concentration in the images corrected for dilution. We call the attention of our readers to the fact that the size of the aperture used to measure central mid-IR fluxes in this paper is different from that used in Paper I. In Paper I, since we did not have the constraint to match the mid-IR aperture to that available for data taken at a different wavelength, the size of the central regions was measured on non-deconvolved mid-IR surface brightness profiles. The aperture sizes given in Table 1 are thus different from those used in Paper I.

Typical errors on disk fluxes are expected to be of the order of 20 or $30 \%$, mainly due to the camera memory effects $^{1}$ and to flux calibration uncertainties.

\section{The star formation rate scaling in galactic disks}

In M 51 and NGC 7331 for example, there exists a striking correspondence between the spatial distributions of $\mathrm{H} \alpha$ and $15 \mu \mathrm{m}$ emission (Sauvage et al. 1996; Smith 1998). When we compare in detail our 7 and $15 \mu \mathrm{m}$ maps with the $\mathrm{H} \alpha$ maps at our disposal, all structures, such as rings, bars and arms, are very similar at the three wavelengths, even without correction for the different angular resolutions and sensitivities. Thus, it is tempting to check the robustness of this correlation in a more quantitative approach. If it holds for total fluxes, it would indicate a strong relationship between mid-infrared dust emission and the SFR.

We first have to cancel identifiable bias sources in our data. In addition to the varying contribution of the cen-

\footnotetext{
1 The stabilization correction that we applied uses the latest available technique taking into account the detector characteristics, described in Coulais \& Abergel (2000).
}

tral regions, the most obvious one is a scale effect, i.e. large - or bright - galaxies tend to be bright at all wavelengths, and comparing them with small and faint galaxies introduces artificial correlation of the data. Another bias results from the use of luminosities instead of fluxes: an incorrect estimation of distances introduces dispersion and the presence of the distance squared on both axes also produces an artificial correlation. We therefore have to normalize our data by another galaxy property, independent of both mid-infrared and $\mathrm{H} \alpha$ emission. We chose for such a quantity the disk area (from the major diameter at the blue isophote $\mu_{B}=25$ mag $\operatorname{arcsec}^{-2}$, defined in the RC3). We stress that even though the normalization of fluxes by the disk area gives quantities that are formally surface brightnesses, these should not be identified with mid-infrared or $\mathrm{H} \alpha$ surface brightnesses. The normalization is only used to avoid the scale-effect in our sample. In the following, we will refer to the quantities obtained in this way as "size-normalized" fluxes.

We applied two fitting procedures. The first one is the classical minimization of squared distances to a line, with an equal treatment of both variables. The second one is the minimization of absolute values instead of squares, again bivariate, and is more robust to outlier points. Figure 1a shows the dependence of the size-normalized $15 \mu \mathrm{m}$ on the size-normalized $\mathrm{H} \alpha$ fluxes. The best least squares fit implies that $F_{15}$ scales as $F(\mathrm{H} \alpha)^{1.01}$, with a correlation coefficient of 0.91 ; the $3 \sigma$ interval for the slope is [0.81; 1.25]. The results for $7 \mu \mathrm{m}$ are quite similar to those for $15 \mu \mathrm{m}$ (Fig. 1b), with approximately the same power law. That the 7 and $15 \mu \mathrm{m}$ flux densities appear interchangeable in Fig. 1 may come at first as a surprise given the already growing amount of literature data indicating that regions of high star formation activity appear as regions of enhanced $15 \mu \mathrm{m}$ emission with respect to $7 \mu \mathrm{m}$ (Vigroux et al. 1996; Sauvage et al. 1996; Dale et al. 2000). However it is also clear that: (1) not all star forming regions show a $15 \mu \mathrm{m}$ excess (see e.g. the color data in Dale et al. 2000, and our Atlas) and (2) the excess appears only above a certain threshold in star-formation activity. This last point is amply demonstrated by the so-called ISO-IRAS colorcolor diagram (Vigroux et al. 1999; Helou 2000) that plots the $F_{15} / F_{7}$ ISO color versus the $F_{60} / F_{100}$ IRAS color: for most of the $F_{60} / F_{100}$ range, the $F_{15} / F_{7}$ color is nearly constant and around 1 , and only starts to increase for the hottest $F_{60} / F_{100}$ colors, corresponding to starburst and interacting galaxies. For normal spiral galaxies such as those in our sample, the $F_{15} / F_{7}$ color does not significantly deviate from 1 . In fact, we show in Paper I that changes of the global $F_{15} / F_{7}$ color of spirals are strictly due to the circumnuclear regions which are not included in Fig. 1. Spiral disks exhibit a $F_{15} / F_{7}$ color of $0.89 \pm 0.14$ (in flux density units, i.e. in Jy).

The fact that the 7 and $15 \mu \mathrm{m}$ fluxes show similar variations with radiation density (in disks) is somewhat puzzling, as they were originally thought to behave quite differently, since the main emission sites of aromatic bands and VSGs (respectively photodissociation regions 
Table 1. Photometric data. Galaxies are named according to the VCC catalog for the Virgo program and according to the NGC catalog for other programs.

\begin{tabular}{|c|c|c|c|c|c|c|c|c|c|}
\hline name & $\begin{array}{c}(\mathrm{H} \alpha+[\mathrm{NII}])_{\mathrm{TOT}^{a}} \\
\left(\log \mathrm{W} \mathrm{m}^{-2}\right)\end{array}$ & $\begin{array}{c}D_{\mathrm{C}}^{b} \\
(\operatorname{arcsec})\end{array}$ & $\begin{array}{c}(\mathrm{H} \alpha+[\mathrm{NII}])_{\mathrm{CEN}}{ }^{a} \\
\left(\log \mathrm{W} \mathrm{m}^{-2}\right)\end{array}$ & $f_{\mathrm{C} 15}{ }^{c}$ & $f_{\mathrm{C} 7^{c}}$ & $F_{15 \text { DISK }}{ }_{(\mathrm{mJy}}^{d}$ & $F_{7 \text { DISK }^{d}}$ & \multicolumn{2}{|c|}{ refs $^{e}$} \\
\hline N337 & -14.38 & $4 . \times 4$ & -15.69 & 0.03 & 0.03 & 288.27 & 327.34 & $11 \mathrm{a}$ & 21 \\
\hline N1022 & -14.91 & & & $\approx 1$ & $\approx 1$ & & & 8 & .. \\
\hline N1097 & -13.94 & 45. & -14.27 & 0.76 & 0.60 & 540.84 & 846.37 & 20 & 20 \\
\hline N1365 & -13.74 & 40. & -14.32 & 0.71 & 0.54 & 1306.65 & 1701.55 & 12 & 12 \\
\hline N1433 & -14.67 & 26. & -15.39 & 0.31 & 0.26 & 246.95 & 283.21 & 2 & 2 \\
\hline N1530 ${ }^{(-)}$ & -14.46 & 28. & -15.32 & 0.44 & 0.38 & 337.69 & 356.16 & 14 & 14 \\
\hline N1672 & -13.93 & 32. & -14.40 & 0.58 & 0.48 & 842.58 & 1030.42 & 20 & 20 \\
\hline $\mathrm{N} 4027$ & -14.41 & 4. $\times 4$ & -16.21 & 0.01 & 0.01 & 668.50 & 765.68 & $11 \mathrm{a}$ & 21 \\
\hline N4535 & -14.26 & $\approx 10$ & -15.22 & 0.11 & 0.08 & 1000.65 & 1047.02 & 24 & 13 \\
\hline N4691 & -14.94 & & & $\approx 1$ & $\approx 1$ & & & 6 & .. \\
\hline N4736 & -13.27 & $\approx 10$ & -15.65 & 0.06 & 0.05 & 3970.51 & 3702.07 & 24 & 13 \\
\hline N5194 & -13.33 & 89. & -14.10 & 0.25 & 0.22 & 5969.43 & 6728.27 & 24 & 7 \\
\hline N5236 & -12.87 & 33. & -13.97 & 0.17 & 0.14 & 16737.02 & 15933.28 & $11 \mathrm{~b}$ & 16 \\
\hline N5383 ${ }^{(-)}$ & -14.23 & 22.3 & -14.49 & 0.49 & 0.41 & 169.72 & 205.100 & 18 & 18 \\
\hline N5457 & -13.17 & 35. & -15.18 & 0.02 & 0.02 & 5295.81 & 5920.03 & $11 \mathrm{~b}$ & 22 \\
\hline N6744 $(-)$ & -13.54 & 4. $\times 4$ & -16.95 & 0.01 & 0.01 & 1494.72 & 2414.59 & 17 & 21 \\
\hline N7552 & -14.19 & 21.3 & -14.50 & 0.83 & 0.69 & 475.78 & 574.27 & 5 & 5 \\
\hline V66 (N4178) & -14.59 & $2 . \times 4$. & -17.22 & 0.02 & 0.01 & 178.19 & 225.74 & 24 & 9 \\
\hline V92 (N4192) & -14.39 & $\approx 5$ & -15.61 & 0.06 & 0.03 & 591.66 & 872.36 & 24 & 13 \\
\hline V460 (N4293) & -14.90 & $\approx 12$ & -14.99 & 0.67 & 0.29 & 62.34 & 112.59 & 24 & 13 \\
\hline V692 (N4351) & -15.43 & 8. & -16.18 & 0.12 & 0.12 & 40.28 & 46.48 & 24 & 19 \\
\hline V836 (N4388) & -14.50 & 12. & -14.97 & 0.73 & 0.38 & 267.86 & 308.83 & $11 \mathrm{~b}$ & 4 \\
\hline V857 (N4394) & -14.96 & $\approx 10$ & -16.56 & 0.09 & 0.09 & 127.13 & 147.36 & 24 & 13 \\
\hline V912 (N4413) & -15.13 & & & & & 92.98 & 89.25 & 24 & .. \\
\hline V1043 (N4438) & -14.61 & 8. & -15.28 & 0.29 & 0.20 & 149.30 & 186.32 & 24 & 19 \\
\hline V1110 (N4450) & -15.17 & $\approx 10$ & -15.68 & 0.11 & 0.09 & 150.36 & 167.55 & 24 & 13 \\
\hline V1379 (N4498) & -15.01 & 8. & -16.53 & 0.05 & 0.05 & 89.63 & 107.73 & 24 & 19 \\
\hline V1673 (N4567) & -14.93 & 8. & -16.20 & 0.05 & 0.04 & 279.19 & 306.28 & 24 & 19 \\
\hline V1676 (N4568) & -14.53 & 8. & -15.88 & 0.08 & 0.06 & 1016.44 & 1014.13 & 24 & 19 \\
\hline V1690 (N4569) & -14.22 & 8. & -15.21 & 0.19 & 0.08 & 758.34 & 776.63 & 24 & 19 \\
\hline V1727 (N4579) & -14.45 & $\approx 10$ & -14.93 & 0.17 & 0.07 & 513.84 & 624.93 & 24 & 13 \\
\hline V1972 (N4647) & -14.66 & 8.1 & -16.09 & 0.04 & 0.03 & 454.66 & 459.85 & 24 & 10 \\
\hline V1987 (N4654) & -14.32 & $\approx 10$ & -15.64 & 0.05 & 0.05 & 962.89 & 1000.25 & 15 & 13 \\
\hline V2058 (N4689) & -14.78 & 8. & -16.83 & 0.02 & 0.02 & 323.91 & 335.34 & $11 \mathrm{a}$ & 19 \\
\hline N986 & -14.47 & 17.8 & -15.00 & 0.57 & 0.36 & 454.91 & 512.01 & 8 & 8 \\
\hline N1326 & -14.71 & 26. & -14.78 & 0.85 & 0.69 & 42.17 & 87.09 & 2 & 2 \\
\hline N1385 & -14.31 & $4 . \times 4$ & -15.93 & 0.02 & 0.01 & 768.69 & 804.42 & $11 \mathrm{a}$ & 21 \\
\hline N3885 & -14.96 & 14.8 & -15.13 & 0.75 & 0.75 & 99.60 & 85.69 & 8 & 8 \\
\hline N4041 & -14.25 & & & & & 751.86 & 792.81 & 24 & .. \\
\hline $\mathrm{N} 4102$ & -14.43 & $34.4 \times 34.4$ & -14.66 & 0.88 & 0.69 & 207.48 & 254.07 & 1 & 1 \\
\hline N4519 & -14.74 & 8. & -16.31 & 0.28 & 0.08 & 168.59 & 162.81 & 24 & 19 \\
\hline N4713 & -14.49 & & & & & 209.41 & 223.64 & 15 & .. \\
\hline N5962 & -14.62 & 4. $\times 4$ & -16.50 & 0.02 & 0.01 & 500.70 & 479.09 & $11 \mathrm{a}$ & 21 \\
\hline N6753 & -14.63 & & & & & 646.77 & 586.44 & 2 & .. \\
\hline N6946 ${ }^{(-)}$ & -12.98 & 45. & -13.70 & 0.19 & 0.11 & 8640.46 & 10401.74 & 23 & 3 \\
\hline N7218 & -14.75 & & & & & 273.49 & 260.59 & $11 \mathrm{a}$ &.. \\
\hline
\end{tabular}

${ }^{a}$ Corrected for Galactic extinction using the RC3 blue absorptions and the extinction curve of Cardelli et al. (1989). Total pure $\mathrm{H} \alpha$ fluxes, available for N1530, N5383, N6744 and N6946, were made homogeneous with (H $\alpha+[$ NII]) fluxes by applying a factor $4 / 3$.

${ }^{b}$ Diameter aperture or slit dimensions of the measurement used to remove a central $\mathrm{H} \alpha$ contribution.

${ }^{c}$ Approximate fractions of central mid-infrared fluxes inside the same aperture as used for $\mathrm{H} \alpha$ (corrected for dilution effects).

${ }^{d}$ Mid-infrared fluxes after removal of the central contribution matched to the $\mathrm{H} \alpha$ aperture.

${ }^{e}$ Reference codes for the total $\mathrm{H} \alpha$ flux and the central $\mathrm{H} \alpha$ flux.

(1) Armus et al. (1990); (2) Crocker et al. (1996); (3) Engargiola (1991); (4) Falcke et al. (1998); (5) map provided by C. Feinstein; (6) map provided by J. A. García-Barreto; (7) map taken from the electronic edition of Greenawalt et al. (1998); (8) Hameed \& Devereux (1999); (9) Ho et al. (1997); (10) Keel et al. (1983); (11a) Kennicutt \& Kent (1983); (11b) Kennicutt et al. (1994); (12) map provided by M. Naslund; (13) Pogge (1989); (14) map provided by M. Regan; (15) Romanishin (1990); (16) map provided by S. D. Ryder; (17) Ryder \& Dopita (1994); (18) Sheth (2000); (19) Stauffer (1983); (20) map provided by T. Storchi-Bergmann; (21) Véron-Cetty \& Véron (1986); (22) map provided by F. Viallefond; (23) Wang (1999); (24) Young et al. (1996).

The correction for stellar absorption of Ho et al. (1997) was cancelled, using their data.

${ }^{(+)}$close system : we used the total $\mathrm{H} \alpha$ flux listed by Young et al. (1996) weighted by the two contributions inside a smaller aperture derived from Kennicutt et al. (1987).

${ }^{(-)}(\mathrm{H} \alpha+[\mathrm{NII}])$ flux estimated from a pure $\mathrm{H} \alpha$ flux. 

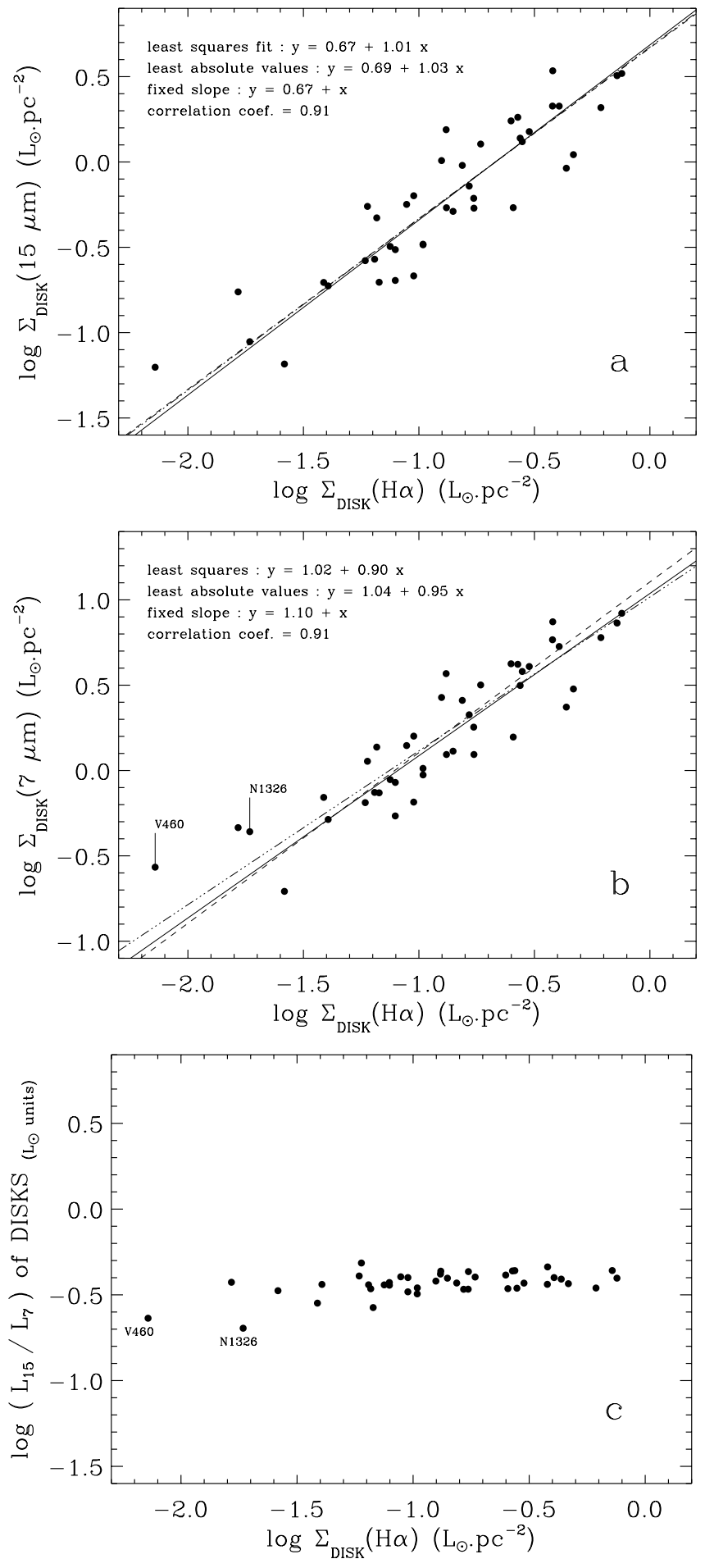

Fig. 1. Relationship between size-normalized fluxes of $\mathrm{H} \alpha$ and: a) $F_{15}(12$ to $18 \mu \mathrm{m})$; b) $F_{7}(5$ to $8.5 \mu \mathrm{m})$. The dashed line is the linear correlation. The best least squares fit and least absolute deviation fit are shown as dot-dashed and solid lines. NGC 4736 and NGC 6744 were excluded because their disk was not completely mapped at 7 and $15 \mu \mathrm{m}$. Excluding in b) the two galaxies with suspected significant contribution from stellar emission at $7 \mu \mathrm{m}$, because of their low $F_{15} / F_{7}$ ratios (VCC 460 and NGC 1326), the fitted slopes become 1.00 and 1.07 , instead of 0.90 and 0.95 . c) shows that $F_{15} / F_{7}$ is constant with a good approximation in disks. Flux density ratios (Jy units) can be obtained applying a factor 2.40 (0.38 dex). and Hir regions) are distinct. A likely explanation is that when averaged in disks, the $15 \mu \mathrm{m}$ emission is no longer dominated by VSGs as in His regions but by a part of the feature composed of the aromatic bands at 11.3 and $12.7 \mu \mathrm{m}$, and thus shares a common origin with the $7 \mu \mathrm{m}$ emission. This is what can be seen in our few mid-IR spectra (Paper I). Other fainter UIBs are additionally present in the $13-18 \mu \mathrm{m}$ range (see for instance Hony et al. 2001). A further confirmation of the common origin of the 7 and $15 \mu \mathrm{m}$ fluxes in disks can be found in the fact that the dependence of $F_{15} / F_{7}$ on the IRAS color $F_{25} / F_{12}$ is weak for low ratios $\left(F_{25} / F_{12}<2\right)$ and begins to strengthen only for relatively hot colors: as the $25 \mu \mathrm{m}$ flux density is clearly due to VSGs, this indicates another origin for the $15 \mu \mathrm{m}$ flux density in the low $F_{25} / F_{12}$ range.

Figure 1 thus implies that on the scale of galactic disks, in the mid-infrared, Hir regions are seen only through their effect of globally increasing the interstellar radiation field. Since impulsive heating of aromatic band carriers by single photons causes the shape of the spectrum to be very insensitive to the radiation intensity (Boulanger et al. 1996), this explains the constant $F_{15} / F_{7}$ ratio observed in our sample. It results from this that even over the large range in Hir region sizes and densities seen along the Hubble sequence, what we observe in disks is mainly the emission from photodissociation regions, and the filling factor by Hit regions is always comparatively small.

Although mid-IR emission can in principle originate from regions where old stars dominate, when integrated throughout spiral disks, the emission in both bandpasses traces young stars and the heating provided by a more evolved population appears negligible. Otherwise, the $F_{15} / \mathrm{H} \alpha$ ratio would increase with decreasing SFR surface density, as the importance of the diffuse interstellar medium relative to star forming regions is then higher; this is not observed, and neither is a variation of $F_{15} / \mathrm{H} \alpha$ with Hubble type.

An alternative cause of the observed correlation could be a much more indirect link between star formation and dust emission, both of them being causally associated with the molecular gas phase (dust is mixed with gas, and stars form out of molecular clouds), and these two links then simulate a direct connection between the presence of massive stars and dust excitation. We have no means of deciding which scenario is the more likely, and they would observationally be extremely difficult to test, in particular because molecular gas mass estimates are not accurate enough. Nevertheless, the presence of molecular gas is a prerequisite but certainly not a sufficient condition for star formation, so that the link between the two is not more direct than the link between young stars and dust emission.

Assuming a purely linear correlation between UIB and $\mathrm{H} \alpha$ emission (as indicated in Fig. 1 by the dashed line) and using the $\mathrm{H} \alpha$-SFR calibration of Kennicutt (1998a) leads to the following scalings:

$$
\begin{aligned}
\operatorname{SFR}\left(M_{\odot} \mathrm{yr}^{-1}\right) & =6.5 \times 10^{-9} L\left(15 \mu \mathrm{m}_{\mathrm{UIB}}\right)\left(L_{\odot \mathrm{bol}}\right) \\
& =2.4 \times 10^{-9} L\left(7 \mu \mathrm{m}_{\mathrm{UIB}}\right)\left(L_{\odot \mathrm{bol}}\right)
\end{aligned}
$$




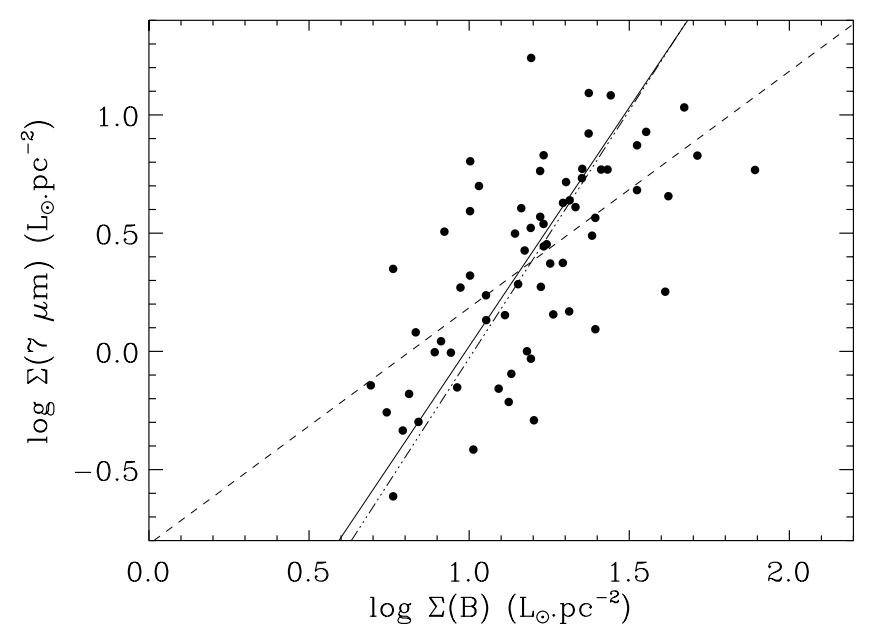

Fig. 2. Comparison of total size-normalized fluxes in the blue band and at $7 \mu \mathrm{m}$. The dashed line is the linear correlation, and the best least squares fit and best least absolute deviation fit are shown as dot-dashed and solid lines. The fitted slopes are 2.10 and 2.02 , with a $1 \sigma$ confidence interval $[1.85 ; 2.41]$ and a linear correlation coefficient of 0.65 . Using $15 \mu \mathrm{m}$ fluxes instead of $7 \mu \mathrm{m}$ fluxes or $H$-band fluxes for the stellar emission leads to very similar results. Restricting the sample to the galaxies present in Fig. 1 or to the galaxies dominated by disk emission at $15 \mu \mathrm{m}$ by more than $70 \%$ also produces similarly dispersed distributions with fitted slopes always above 1.6.

with $L_{\odot \text { bol }}=3.827 \times 10^{26} \mathrm{~W}$ (this assumes solar abundances, and a Salpeter IMF ranging from 0.1 to $\left.100 M_{\odot}\right)$. Flux densities at 7 and $15 \mu \mathrm{m}$ were converted into luminosities using bandpasses of $16.18 \mathrm{THz}$ and $6.75 \mathrm{THz}$ respectively. These formulas are applicable only when the mid-infrared emission is dominated by UIBs, with a negligible VSG continuum, which is the case in disks of galaxies, but is not always verified at $15 \mu \mathrm{m}$ (in galactic central regions, for instance).

In our sample, size-normalized SFRs in disks (we mean here physical regions defined in Paper I) range between about 0.3 and $20 M_{\odot} \mathrm{kyr}^{-1} \mathrm{kpc}^{-2}$, from VCC 1043 to NGC 5236, and SFRs of disks between about 0.12 and $8 M_{\odot} \mathrm{yr}^{-1}$.

We emphasize that the relationship found between dust emission and the $\mathrm{H} \alpha$ recombination line is unique and is not reproduced if the $\mathrm{H} \alpha$ emission is replaced by an observable tracing more evolved stellar populations, such as the blue luminosity, coming from the RC3 (Fig. 2).

\section{Discussion}

\subsection{Integrated versus local quantities}

The above calibration, derived from integrated disk fluxes, should not be applied locally in small regions of disks, for several reasons:

- In the diffuse interstellar medium, far away from any star-forming site, a substantial part of UIB carrier heating may be provided by optical photons, since it has been demonstrated, for instance in reflection nebulæ, that this type of heating can be efficient (Uchida et al. 1998). Looking at quantities integrated over large spatial scales, we average in our beam all the stellar populations contributing to the excitation of dust. Since dust heating by ionizing photons is much more efficient than by near-ultraviolet or optical photons, a small population of massive stars mixed with a large population of low-mass stars can still dominate dust heating as soon as it exceeds a certain threshold, still to be determined. It is clear that this could no longer be the case in selected regions of galaxies.

- Close to Hir regions and in some giant star formation complexes, the $F_{15} / F_{7}$ color is observed to rise above the mean value in disks (see for instance NGC 5457 in the atlas), revealing a thermodynamical state different from that in disks for the species emitting at these two wavelengths. This $15 \mu \mathrm{m}$ excess clearly breaks down the symmetry of the 15 and $7 \mu \mathrm{m}$ fluxes, and then most likely the validity of our calibration.

- A significant fraction of ionizing photons is able to escape His regions, and possibly to propagate to very large distances. In such a configuration, a physical link between young stars and dust heating can be established only using integrated fluxes.

To support the last caveat, we note that Beckman et al. (2000) propose, from their study of $\mathrm{H} \alpha$ luminosity functions of Hir regions, that the estimated ionizing luminosity able to escape the most luminous Hir regions is more than sufficient to ionize the warm diffuse interstellar medium (see also Oey \& Kennicutt 1997 and references therein); it is hence also able to heat dust to comparable distances. If the idea that the diffuse ionized gas is excited by the photons escaping from Hir regions is confirmed, then the high $\mathrm{H} \alpha$ luminosity fractions attributable to this diffuse medium (30-50\% according to e.g. Ferguson et al. 1996) imply that a correct assessment of star formation activity cannot be made if restricted to limited regions, neither using recombination lines nor using the infrared emission.

Vogler et al. (2001) have looked at the relationship between $\mathrm{H} \alpha$ and $7 \mu \mathrm{m}$ fluxes locally inside the disk of NGC 5236, using resolution elements of $12^{\prime \prime}(\approx 300$ pc at our adopted distance). They find a very large dispersion, mostly at low flux levels, so that the correlation that we report here breaks down at kpc-scales. It is however difficult to disentangle in the observed scatter the role played by variable extinction in the $\mathrm{H} \alpha$ line (spatially and also as a function of brightness) from a physical decorrelation.

\subsection{Mid-IR versus far-IR emission as tracers of star formation}

In Sect. 3, restricting ourselves to spiral disks, we have found that mid-IR luminosities can be used to trace the level of star formation. As mentioned in Sect. 1, numerous attempts to use the far-IR luminosity as a tracer of star 


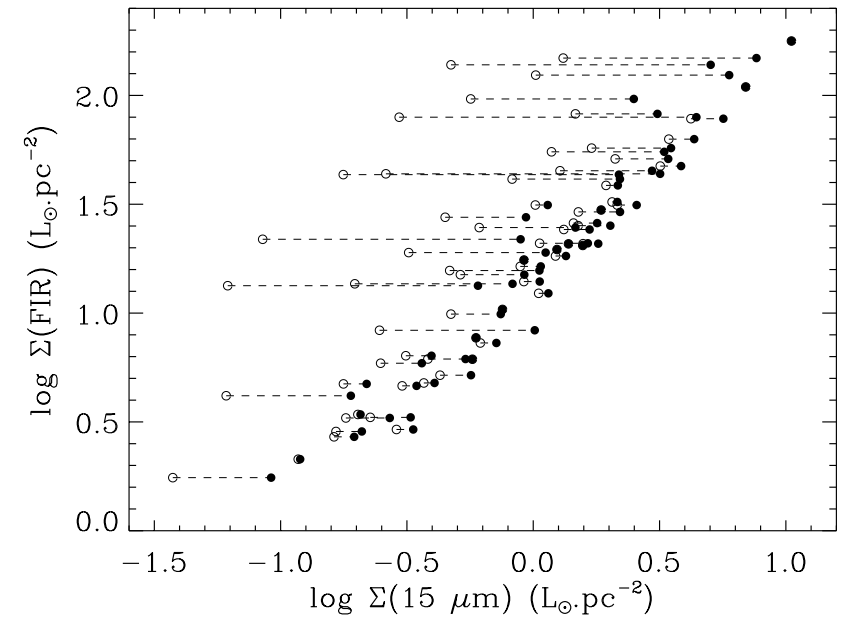

Fig. 3. Proportionality between far-infrared and mid-infrared size-normalized fluxes. Far-IR fluxes are a combination of 60 and $100 \mu \mathrm{m}$ IRAS fluxes as defined in Helou et al. (1988). Filled circles represent total fluxes and open circles show the effect of taking into account only bare disks, as defined in Paper I (this was possible only at $15 \mu \mathrm{m}$, since galaxies are not resolved by IRAS). The best least-squares fit gives a slope of $1.08\left({ }_{-0.10}^{+0.11}\right.$ at a $3 \sigma$ confidence level) with a correlation coefficient of 0.97 . The relationship with $7 \mu \mathrm{m}$ fluxes is similar, but more dispersed and with a slightly higher slope $\left(1.20_{-0.15}^{+0.18}\right.$ at a $3 \sigma$ confidence level).

formation have already been presented (see e.g. Devereux \& Young 1990; Kennicutt 1998a and references therein). It is thus worthwhile to examine whether the tracer we propose here presents some significant advantage over the far-IR one. The main problem with the far-IR luminosity is that the heating radiation required for grains to emit in that wavelength range can easily be provided by relatively old stars of $10^{8} \mathrm{yr}$ or more (Buat \& Xu 1996) and thus the information derived on the SFR is different from that obtained from $\mathrm{H} \alpha$. In other terms, when plotting far-IR versus $\mathrm{H} \alpha$ data, there is a hidden variable which corresponds to the amount of energy provided by non-ionizing stars. It is this hidden variable which has previously been deemed responsible of the significant non-linearity of the far-IR$\mathrm{H} \alpha$ correlation (e.g. Lonsdale-Persson \& Helou 1987).

Figure 3 shows that the two integrated far-IR and $15 \mu \mathrm{m}$ size-normalized fluxes are tightly and linearly correlated. Consequently, there cannot be major differences in the heating source for the small grains responsible for the $15 \mu \mathrm{m}$ emission and the large grains emitting in the far-infrared. If we restrict ourselves to galaxies with small circumnuclear contribution (in Fig. 3, those for which the two symbols used are close to each other), this suggests that in galaxies which are dominated by their disk, the far-infrared emission is an equivalently good star formation tracer as the 15 and $7 \mu \mathrm{m}$ emission.

In Fig. 4, we plot the correlation between the sizenormalized total far-IR and $\mathrm{H} \alpha$ fluxes for galaxies in our sample. Given the very poor spatial resolution of current far-IR instruments, it is impossible to separate nuclear and disk contributions. We see here that the correlation is

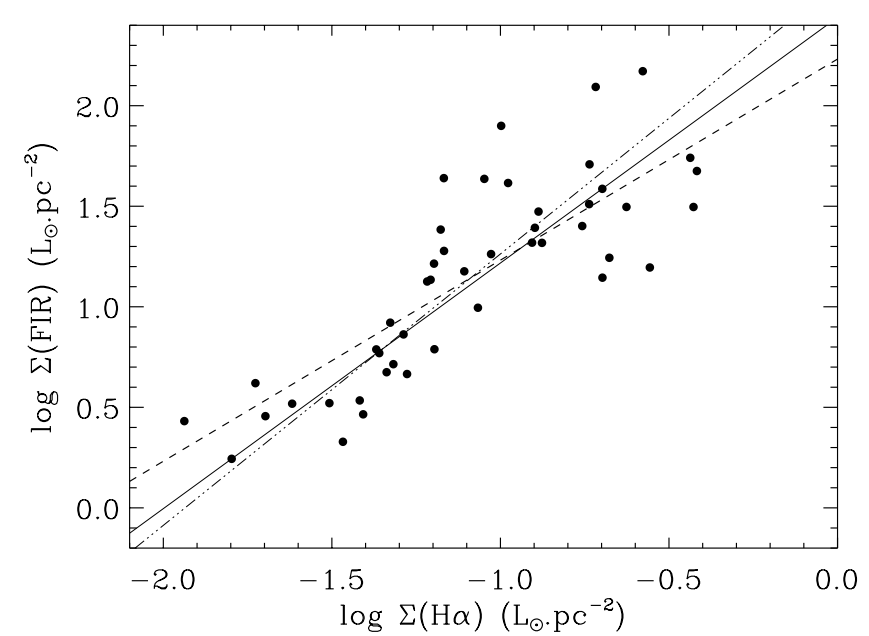

Fig. 4. Relationship between total far-IR and $\mathrm{H} \alpha$ sizenormalized fluxes. The dashed line represents the linear correlation, the dot-dashed line the least squares fits, and the solid line the least absolute deviation fit, with respective slopes $1.35_{-0.34}^{+0.50}(3 \sigma$ interval $)$ and 1.22 .

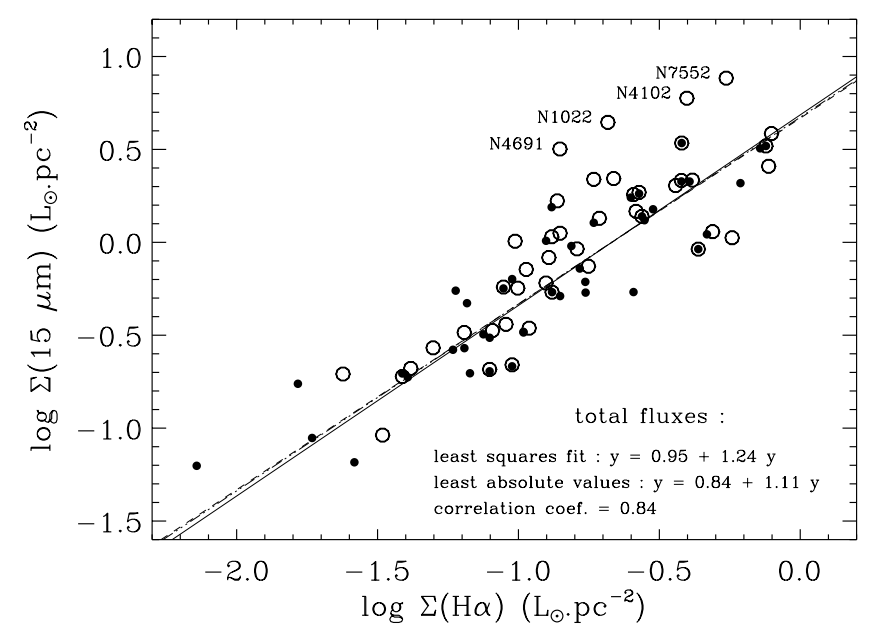

Fig. 5. Same as Fig. 1, with total size-normalized $15 \mu \mathrm{m}$ and $\mathrm{H} \alpha$ fluxes superimposed, shown as empty circles. The solid and dot-dashed lines correspond to the fits on disk fluxes of Fig. 1. The names of the most strongly deviating galaxies and the numerical results of the fits on total fluxes are indicated. At $7 \mu \mathrm{m}$, only NGC 1022 and 4691 tend to stray from the relationship found in disks.

worse than that obtained in Fig. 1 and in particular that the well-known non-linearity of the correlation is found in our sample too: a least-square fit to our data gives a slope of $1.35^{2}$. The results obtained are highly dependent on the adopted references for IRAS fluxes among the various catalogs (Moshir et al. 1989; Rice et al. 1988; Soifer et al. 1989; Helou et al. 1988; Sanders et al. 1995; Rush et al. 1993; Thuan \& Sauvage 1992; PSC), which may partially account for the dispersion. However, we have tried to identify the most reliable fluxes by optimizing the agreement

\footnotetext{
${ }^{2}$ Using a large sample drawn from the $\mathrm{H} \alpha$ catalogues mentioned in Sect. 2 and applying a robust estimation method to reduce the effects of outlying points, since a significant scatter is present, we obtain $\Sigma\left(F_{\mathrm{FIR}}\right) \propto \Sigma(\mathrm{H} \alpha)^{1.43}$.
} 
between our 7 and $15 \mu \mathrm{m}$ fluxes and IRAS $12 \mu \mathrm{m}$ fluxes, whose bandpass overlaps with both ISOCAM bandpasses.

Looking for the origin of this non-linearity, we find that it is mainly introduced in our sample by prominent circumnuclear regions. Indeed, when the fraction of the $15 \mu \mathrm{m}$ flux contributed by central regions increases (from nearly 0 to nearly 1$)$, the mean $F_{\mathrm{FIR}} / F(\mathrm{H} \alpha)$ ratio rises from $\approx 130$ to $\approx 480$. The increase of $F_{\mathrm{FIR}} / F(\mathrm{H} \alpha)$ is also connected with the appearance of high $F_{15} / F_{7}$ colors, which have been shown in Paper I to originate in central regions of galaxies whose total mid-infrared emission is dominated by a central starburst. We can further check this finding by estimating the fraction of far-IR emission arising from the disk alone and producing an analog of Fig. 1 for the far-IR. Since no spatially-resolved measurements are available, we have to rely on disk flux fractions measured at $15 \mu \mathrm{m}$ and assume that they can account for the true fractions in the far-IR. Given that total $15 \mu \mathrm{m}$ and far-IR fluxes are tightly correlated, this assumption is sensible. This empirical correction succeeds in linearizing the far-IR-H $\alpha$ relationship and reducing the scatter: the least squares and least absolute deviation fits give a slope of respectively $1.01_{-0.20}^{+0.25}$ and 1.07 . This demonstrates that it is the circumnuclear contribution which creates the nonlinearities observed in previous attempts to correlate the far-infrared and $\mathrm{H} \alpha$ fluxes.

To further support this view, we have looked at correlations between total mid-infrared and $\mathrm{H} \alpha$ fluxes, adding the two galaxies whose mid-infrared and $\mathrm{H} \alpha$ emission is highly concentrated, NGC 1022 and 4691. Figure 5 shows the result at $15 \mu \mathrm{m}$, compared with what we have previously obtained in disks. Owing to the fact that $\mathrm{H} \alpha$ data were found for only few centrally dominated galaxies with a $15 \mu \mathrm{m}$ excess, the relationship for total fluxes is not much different from that for disk fluxes. However, the fits indicate that the dispersion is already higher and that we introduced a non-linearity. As the extinction in circumnuclear regions is expected to be higher than in the average disk, the interpretation of the cause for the non-linearity is not straightforward. We postpone the detailed study of mid-infrared emission and other star formation estimates in circumnuclear and starburst regions (Förster-Schreiber \& Roussel, in preparation), and outline that the relationship found here is strictly valid only in disks, and cannot be extrapolated easily.

\subsection{Applicability at high redshift}

In cosmological surveys with the HST, SFRs are measured with fluxes that correspond to ultraviolet wavelengths in the rest-frame of distant galaxies. For this reason, we have tried to compare the SFRs deduced from mid-infrared fluxes using Eq. (1) with the SFRs derived from ultraviolet fluxes at $1650 \AA$ using the calibration given in Kennicutt (1998a). The data come from Rifatto et al. (1995), Deharveng et al. (1994) and Bell \& Kennicutt (2001) (whose filters are not centered exactly at $1650 \AA$ but at 1615 and $1521 \AA$ ), and the sample was limited to galaxies either with a disk flux fraction above 0.7 or with $F_{15} / F_{7} \leq 1.2$ (respectively 20 and 29 objets), both criteria leading to the same conclusion. UV fluxes have only been corrected for Galactic extinction in the same way as $\mathrm{H} \alpha$, and the internal extinction has been estimated by the requirement that the 7 or $15 \mu \mathrm{m}$ and ultraviolet SFR values agree for each spiral. Resulting UV absorptions range between 0.2 and $2.6 \mathrm{mag}$, and their median value amounts to $1.4-1.7 \mathrm{mag}$. We recall that our calibration assumes an $\mathrm{H} \alpha$ absorption of $1.1 \mathrm{mag}$, and that changing $A(\mathrm{H} \alpha)$ would change $A_{1650}$ by the same amount.

We can compare these numbers with those obtained in the high-redshift sample of Flores et al. (1999) $(0.2<z<$ 1.1), who have performed a similar comparison between star formation rates derived from ultraviolet and infrared observations. They have extrapolated ultraviolet fluxes at $2800 \AA$ from spectral energy distributions above $4350 \AA$, using a grid of spectro-photometric evolutionary models, and have also estimated total infrared fluxes from 8 to $1000 \mu \mathrm{m}$ from mid-infrared and radio fluxes, fitted by a set of templates. Assuming the same IMF as in the present work and the SFR calibrations of Kennicutt (1998a) both in terms of UV and IR fluxes, Flores et al. (1999) derive extinctions in the range $0.5 \leq A_{2800} \leq 2.2 \mathrm{mag}$. This translates into $0.6<A_{1650}<2.8$, using the extinction curves of Cardelli et al. (1989). Absorption estimates in our sample are thus consistent, well within the uncertainties, with those in the sample of Flores et al. (1999). Hence, although absorption estimates can be flawed by metallicity effects in young galaxies - if they deplete preferentially small carbonaceous grains emitting in the mid-infrared there is no hint of a significant increase of optical depths with redshift, which implies that the calibration we give likely remains valid in more distant galaxies.

\section{Summary and conclusions}

We have seen that mid-infrared fluxes can be considered reliable tracers of star formation in relatively quiescent environments such as spiral galactic disks. There, midinfrared spectra between 5 and $18 \mu \mathrm{m}$ are dominated by aromatic bands, which are much more tightly linked with the radiation from young stars than with the blue-band radiation, to which stars of all masses and ages contribute.

The calibration in terms of SFR that we propose depends of course on the adopted IMF, but most sensitively on the extinction correction applied to $\mathrm{H} \alpha$ fluxes. The dispersion in $\mathrm{H} \alpha$ fluxes with respect to the linear correlation is 0.19 dex in Fig. 1, much lower than the extinction correction of 0.44 dex. We find a bivariate dispersion around the linear correlation of a factor of 1.37 and a one-dimensional dispersion of a factor of 1.56 , both at 7 and $15 \mu \mathrm{m}$. Despite this large scatter, it is meaningful to apply our calibration to large samples, because the sizenormalized SFR shows a much larger range (it varies by more than a factor of 50 across the present sample). 
Nevertheless, one must be aware of several limitations:

- Because dust grains can be heated by the radiation from His regions at large distances from them, and because dust, in some diffuse regions, can be predominantly heated by old stellar populations, the relationship between mid-infrared fluxes and star formation rates is certainly much more complex locally than when considering integrated fluxes.

- For distant objects detected in surveys, the only available information consists of fluxes integrated over the whole galaxy, which can be dominated by the central regions. In this case, the link between star formation and mid-infrared emission can also be completely different. Indeed, we show that considering global fluxes, in the far-infrared as well as at $15 \mu \mathrm{m}$, introduces a non-linearity in the correlation with $\mathrm{H} \alpha$ fluxes. This may be due to a combination of several effects: a thermodynamical state of dust grains in central regions different from that in disks; a greater extinction affecting the $\mathrm{H} \alpha$ line; a dominant contribution from post-starburst populations of the bulge to dust heating in the absence of significant star formation.

- Our sample comprises only spiral galaxies whose metallicity is thought to be near solar, while metaldeficiency (mostly seen in blue compact and dwarf irregular galaxies) can alter the dust composition and is likely to deplete carbonaceous grains, which tends to lower the mid-infrared emission for a given radiation field (Sauvage et al. 1990; Boselli et al. 1998). The aromatic bands are often absent or very weak in dwarf galaxies, due to the above effect combined with their destruction by the far-ultraviolet radiation, very pervasive in low-metallicity environments (Madden 2000).

- In extremely active environments with normal metallicity, the aromatic band carriers can also be destroyed or experience chemical transformations, but quantitative estimations of these effects are yet unavailable.

However, this relationship between mid-IR fluxes and SFRs that strictly holds only in normal disks can be useful to interpret surveys made in the two filters LW3 $(15 \mu \mathrm{m})$ and LW2 $(7 \mu \mathrm{m})$. For galaxies at high redshifts $(z \simeq 1.2)$, the LW2 rest frame emission is shifted to the LW3 bandpass. Hence, the calibration given here must provide a lower limit for the true SFR since, for galaxies with greater star formation activity than in the present sample, the energy redistribution favors the LW3 band, as more energy is reradiated by VSGs. Indeed, Boulanger et al. (1998b) have presented observations in resolved Galactic regions (a diffuse cloud and four photodissociation regions) which show that the emission in UIBs tends to rise with the ultraviolet energy density, linearly at low energy densities and more slowly at higher values. The threshold for this transition, above $10^{3}$ times the energy density in the solar neighborhood, is uncertain due to dilution effects. However, the same type of behavior must hold in integrated galaxies.

Dealing with integrated fluxes, a simple validity criterion of the formula presented here would be a rest-frame color $F_{15} / F_{7} \simeq 1$, i.e. the contribution from the central concentration to the total emission should be low or should arise from heating by a disk-like (non-starburst) stellar population.

Acknowledgements. We warmly thank Antonio GarcíaBarreto, Thaisa Storchi-Bergmann, Magnus Naslund, Carlos Feinstein, Michael Regan, Stuart Ryder and François Viallefond for freely providing their $\mathrm{H} \alpha$ maps. We wish to thank Suzanne Madden for taking part in the improvement of the manuscript and Hervé Aussel for a discussion about high redshift studies of star formation.

The ISOCAM data presented in this paper were analyzed using and adapting the CIA package, a joint development by the ESA Astrophysics Division and the ISOCAM Consortium (led by the PI C. Cesarsky, Direction des Sciences de la Matière, CEA, France).

\section{References}

Armus, L., Heckman, T. M., \& Miley, G. K. 1990, ApJ, 364, 471

Beckman, J. E., Rozas, M., Zurita, A., Watson, R. A., \& Knapen, J. H. 2000, AJ, 119, 2728

Bell, E. F., \& Kennicutt, R. C. 2001, ApJ, 548, 681

Binette, L., Magris, C. G., Stasinśka, G., \& Bruzual, A. G. 1994, A\&A, 292, 13

Boselli, A., Lequeux, J., Sauvage, M., et al. 1998, A\&A, 335, 53

Boulade, O., Sauvage, M., Altieri, B., et al. 1996, A\&A, 315, L85

Boulanger, F., Reach, W. T., Abergel, A., et al. 1996, A\&A, 315, L325

Boulanger, F., Boissel, P., Cesarsky, D., \& Ryter, C. 1998a, A\&A, 339, 194

Boulanger, F., Abergel, A., Bernard, J. P., et al. 1998b, ASP Conf. Ser., 132, 15

Brand, P. W. J. L., Coulson, I. M., \& Zealey, W. J. 1981, MNRAS, 195, 353

Buat, V., \& Xu, C. 1996, A\&A, 306, 61

Cardelli, J. A., Clayton, G. C., \& Mathis, J. S. 1989, ApJ, 345, 245

Cesarsky, D., Lequeux, J., Abergel, A., et al. 1996, A\&A, 315, L309

Coulais, A., \& Abergel, A. 2000, A\&AS, 141, 533

Crocker, D. A., Baugus, P. D., \& Buta, R. 1996, ApJS, 105 353

Dale, D. A., Silbermann, N. A., Helou, G., et al. 2000, AJ, 120, 583

Deharveng, J.-M., Sasseen, T. P., Buat, V., et al. 1994, A\&A, 289,715

Désert, F.-X., Boulanger, F., \& Puget, J. L. 1990, A\&A, 237, 215

Devereux, N. A., \& Young, J. S. 1990, ApJ, 350, L25

Engargiola, G. 1991, ApJS, 76, 875

Falcke, H., Wilson, A. S., \& Simpson, C. 1998, ApJ, 502, 199

Feinstein, C. 1997, ApJS, 112, 29 
Ferguson, A. M. N., Wyse, R. F. G., Gallagher, J. S., \& Hunter, D. A. 1996, AJ, 111, 2265

Flores, H., Hammer, F., Thuan, T. X., et al. 1999, ApJ, 517, 148

Förster-Schreiber, N. M., \& Roussel, H. 2001, in preparation

García-Barreto, J. A., Franco, J., Carrillo, R., Venegas, S., \& Escalante-Ramírez, B. 1996, RMxAA, 32, 89

Giard, M., Pajot, F., Lamarre, J. M., Serra, G., \& Caux, E. 1989, A\&A, 215, 92

Greenawalt, B., Walterbos, R. A. M., Thilker, D., et al. 1998, ApJ, 506, 135

Hameed, S., \& Devereux, N. 1999, AJ, 118, 730

Helou, G. 2000, in Infrared astronomy: today and tomorrow, Les Houches Summer School Aug. 1998 (Springer-Verlag), 337

Helou, G., Khan, I. R., Malek, L., \& Boehmer, L. 1988, ApJS, 68, 151

Helou, G. 1986, ApJ, 311, L33

Ho, L. C., Filippenko, A. V., \& Sargent, W. L. W. 1997, ApJS, 112,315

Hony, S., van Kerckhoven, C., Peeters, E., et al. 2001, A\&A, 370,1030

Keel, W. C. 1983, ApJS, 52, 229

Kennicutt, R. C. 1983, ApJ, 272, 54

Kennicutt, R. C., \& Kent, S. M. 1983, AJ, 88, 1094

Kennicutt, R. C., Keel, W. C., van der Hulst, J. M., Hummel, E., \& Roettiger, K. A. 1987, AJ, 93, 1011

Kennicutt, R. C., Tamblyn, P., \& Congdon, C. W. 1994, ApJ, 435, 22

Kennicutt, R. C. 1998a, ARA\&A, 36, 189

Kennicutt, R. C. 1998b, ApJ, 498, 541

Léger, A., \& Puget, J. L. 1984, A\&A, 137, L5

Lemke, D., Mattila, K., Lehtinen, K., et al. 1998, A\&A, 331, 742

Lindblad, P. O. 1999, A\&AR, 9, 221

Lonsdale-Persson, C. J., \& Helou, G. 1987, ApJ, 314, 513

Madden, S. C. 2000, New Astron. Rev., 44, 249

Mattila, K., Lemke, D., Haikala, L. K., et al. 1996, A\&A, 315, L353

Moshir, M., Copan, G., Conrow, T., et al. 1989, IRAS Faint Source Catalog

Oey, M. S., \& Kennicutt, R. C. 1997, MNRAS, 291, 827

Pogge, R. W. 1989, ApJS, 71, 433

Puget, J. L., \& Léger, A. 1989, ARA\&A, 27, 161

Regan, M. W., Teuben, P. J., \& Vogel, S. N. 1996, AJ, 112, 2549

Rice, W., Lonsdale, C. J., Soifer, B. T., et al. 1988, ApJS, 68, 91
Rifatto, A., Longo, G., \& Capaccioli, M. 1995, A\&AS, 114, 527

Romanishin, W. 1990, AJ, 100, 373

Roussel, H., Vigroux, L., Bosma, A., Sauvage, M., et al. 2001a, A\&A, 369, 473 (Atlas)

Roussel, H., Vigroux, L., Sauvage, M., et al. 2001b, 372, 406 (Paper I)

Rush, B., Malkan, M. A., \& Spinoglio, L. 1993, ApJS, 89, 1

Ryder, S. D., \& Dopita, M. A. 1994, ApJ, 430, 142

Ryder, S. D., Hungerford, A., Dopita, M. A., et al. 1995, in The Opacity of Spiral Disks, ed. J. I. Davies, \& D. Burstein (Dordrecht: Kluwer), 359

Sanders, D. B., Egami, E., Lipari, S., Mirabel, I. F., \& Soifer, B. T. 1995, AJ, 110, 1993

Sauvage, M., Thuan, T. X., \& Vigroux, L. 1990, A\&A, 237, 296

Sauvage, M., \& Thuan, T. X. 1992, ApJ, 396, L69

Sauvage, M., Blommaert, J., Boulanger, F., et al. 1996, A\&A, 315, L89

Sellgren, K., Luan, L., \& Werner, M. W. 1990, ApJ, 359, 384

Sheth, K., Regan, M. W., Vogel, S. N., \& Teuben, P. J. 2000, ApJ, 532, 221

Smith, B. J. 1998, ApJ, 500, 181

Smith, B. J., \& Harvey, P. M. 1996, ApJ, 468, 139

Soifer, B. T., Boehmer, L., Neugebauer, G., \& Sanders, D. B. 1989, AJ, 98, 766

Stauffer, J. R. 1983, ApJ, 264, 14

Storchi-Bergmann, T., Wilson, A. S., \& Baldwin, J. A. 1996, ApJ, 460, 252

Sturm, E., Lutz, D., Tran, D., et al. 2000, A\&A, 358, 481

Thuan, T. X., \& Sauvage, M. 1992, A\&AS, 92, 749

Tran, D. 1998, Ph.D. Thesis, University of Paris XI

Uchida, K. I., Sellgren, K., \& Werner, M. 1998, ApJ, 493, L109

Uchida, K. I., Sellgren, K., Werner, M. W., \& Houdashelt, M. L. 2000, ApJ, 530, 817

de Vaucouleurs, G., de Vaucouleurs, A., Corwin, H. G., et al. 1991, Third Reference Cat. of Bright Galaxies (RC3)

Véron-Cetty, M.-P., \& Véron, P. 1986, A\&AS, 66, 335

Verstraete, L., Puget, J. L., Falgarone, E., et al. 1996, A\&A, 315, L337

Vigroux, L., Mirabel, I. F., Altieri, B., et al. 1996, A\&A, 315, L93

Vigroux, L., et al. 1999, in The universe as seen by ISO, October 20-23 1998, Paris, ESA SP-427

Vogler, A., Madden, S., Sauvage, M., et al. 2001, in preparation

Wang, J., Heckman, T. M., \& Lehnert, M. D. 1999, ApJ, 515, 97

Young, J. S., Allen, L., Kenney, J. D. P., Lesser, A., \& Rownd, B. 1996, AJ, 112, 1903 